Research Article

\title{
Life Prediction Method of Remanufactured Machinery Equipment Based on Vibration Signal Feature Extraction
}

\author{
Bin Li $\mathbb{D}$, Le Kui, Jingdong Luo, and Shiyong Chen \\ College of Naval Architecture and Marine Engineering, Guangzhou Maritime University, Guangzhou 510725, China \\ Correspondence should be addressed to Bin Li; purplemuzi@gzmtu.edu.cn
}

Received 8 November 2021; Revised 29 November 2021; Accepted 30 November 2021; Published 20 December 2021

Academic Editor: Miaochao Chen

Copyright (C) 2021 Bin Li et al. This is an open access article distributed under the Creative Commons Attribution License, which permits unrestricted use, distribution, and reproduction in any medium, provided the original work is properly cited.

\begin{abstract}
Mechanical equipment is a key component of mechanical equipment, and its working condition is directly related to the overall performance of mechanical equipment. Accurate evaluation and prediction of the performance degradation trend of mechanical equipment is of great significance to ensure the reliability and safety of the mechanical equipment system. Based on the data of typical faulty equipment, this paper analyzes the energy characteristic parameters of mechanical equipment under different types and degrees of failure in the time domain. Using amplitude spectrum analysis, Hilbert envelope demodulation and wavelet packet decomposition method, and other vibration signal processing methods, preliminary extraction of multiple statistical feature parameters are given. Secondly, in view of the irrelevant and redundant components of multiple statistical parameters, a new method for extracting fault features of mechanical equipment based on variance value and principal component analysis is proposed. This method can effectively classify the fault status of mechanical equipment. The effectiveness of the method is verified by actual equipment signals. After that, the value extracted from the vibration signal of the double-row roller equipment is used as the degradation feature. In order to reduce the influence of irregular characteristics in the vibration signal and simplify the complexity of the vibration signal, the wavelet transform and the support vector machine model are combined, according to the degradation after decomposition. The $95 \%$ confidence interval of the predicted value is also given. The SVM model is established based on data characteristics, and single-step and multistep prediction of equipment degradation trends are carried out. The prediction result shows that, according to the mapping position formula, the distribution of equipment degradation prediction points is obtained, and a $95 \%$ confidence interval based on the distribution of the prediction points is given. Finally, on the basis of completing feature extraction, this paper applies an unsupervised feature selection method. The sensitive characteristics of life prediction and the prediction results of a single SVM model and a neural network model are compared and analyzed at the same time.
\end{abstract}

\section{Introduction}

Equipment in the national economic industries such as machinery, transportation, energy, and metallurgy (especially high-end, large, key electromechanical equipment) are often under high load, variable working conditions, and continuous operation. The structure inevitably has different degrees of failure, which often causes economic losses, waste of resources, environmental pollution, and casualties [1]. The safety and reliability of equipment has an important impact on the national economy and people's livelihood, social stability, and national resources and environment. For these major mechanical equipment, the quality of the equipment can be improved after the optimization of the design and manufacturing process, but it is still difficult to guarantee that there will be no failure during the service process [2]. Due to the high cost and actual installation restrictions, this type of equipment is often operated by a single unit, which is a small sample case, and it also lacks an overall operation and degradation curve, in addition, equipment of the same type and model even under the same working conditions. Due to the different operating environments and complex load effects, the life data has a large discreteness, which brings difficulties to accurately predict the remaining life [3].

With the severe problems of energy shortage and environmental degradation, the machinery manufacturing 
process has been developed to higher parameters and larger device scales. The trend of large-scale production, automation, high-parameter operation, and high-energy storage has caused safety problems for mechanical products [4]. The increase in complexity and uncertainty of mechanical products makes it difficult to determine the physical model. Due to its high cost and small batch size, traditional statistical methods based on large samples are not suitable for the scarcity of such mechanical equipment test samples. How to perform state assessment and life prediction based on the characteristic data under a small sample is a very challenging subject, and related theories and methods are in urgent need of improvement [5]. Mechanical equipment is one of the most basic and fault-prone parts in rotating machinery, and its operating status is of great significance for ensuring the safe and reliable operation of key equipment. Condition monitoring of mechanical equipment, when the equipment has degraded characteristics, accurately judging its failure and predicting its remaining life is very important for the reasonable arrangement of equipment maintenance decisions [6-8].

This article focuses on the important scientific issues in the operation and maintenance of machinery and equipment at this stage, combined with the development plan of the national machinery and manufacturing science, researches the support vector machine model and state space model in the data-driven life prediction method, and uses system science to discover and understand the general law of life prediction of complex equipment based on theories and methods. Based on the vibration signal of mechanical equipment, three aspects are studied: the purpose is to analyze the failure mechanism of mechanical equipment and various statistical characteristics, on this basis, improve the reliability of fault diagnosis, and propose a method for extracting fault characteristics of typical operating components. The predicted value of the remaining life is very close to the true value, which proves the validity of the model. Effectively we identify the failure state of key equipment and mechanical equipment, improve the effectiveness of forecasting, construct relevant datadriven forecasting models, and discuss their effectiveness. This paper takes the vibration signal of mechanical equipment as the research object and studies the two key issues of vibration fault feature extraction and remaining life prediction.

\section{Related Work}

In order to solve the problem of safe and reliable operation of equipment, relevant research work at home and abroad has roughly gone through three stages: the state monitoring of the equipment, mainly to determine whether the equipment is in normal service; and the fault diagnosis of the equipment, mainly to analyze the failure when a fault occurs. Early warning of equipment failure is mainly carried out when there is no failure, and modern maintenance methods such as predictive maintenance are developed on this basis. This is generally aimed at predictable failures, such as time-dependent failures and progressive failures [9-11].

The essence of condition monitoring is to collect information that contains the characteristics of the current state of the equipment and apply vibration signal processing tech- nology to effectively extract the characteristics of the collected vibration signals, which can provide support for equipment fault diagnosis and fault warning, so as to achieve a more scientific equipment maintenance method. Based on the essential attribute that mechanical failure is both a state and a process, Wang et al. [12] use the state parameters and failure signs monitored during equipment operation to realize the dynamic identification of the failure state and evolution process and condense the key sciences in the diagnosis and prediction of mechanical failures. The problems include the theory and method of multifault feature extraction and recognition and the safety assurance technology based on the reliability of state information. He et al. [13] put forward the technical difficulties of equipment fault diagnosis and prediction: the equipment is of multiple types: with complex operating conditions (no-stationary, nonlinear, variable conditions, and long history). Research shows that the more complex the equipment and the state, the lower the failure rate. Li et al. [14] proposed the concept of wavelet packet energy flow based on vibration signals, using the energy matrix of each node of the wavelet packet as feature information, reducing redundant time-frequency information through popular learning, and fully extracting timefrequency features and nonlinearity, which effectively realizes different types of equipment failures of different degrees. The wavelet theory is still developing, and the research of wavelet analysis in the feature extraction of mechanical equipment will continue to deepen.

Wang et al. [15] established a framework for estimating the remaining life of components based on particle filtering. This method avoids the assumptions of linearity and Gaussian noise and achieves good results in crack prediction. The online monitoring data of the compressor is collected as historical data and the state space. The model and particle filter are used to predict the degradation trend. The prediction results show the effectiveness of the method. The degradation model is established by making full use of historical data, and the idea of joint estimation based on the state and static parameters of the particle filter is adopted to obtain the automatically updated results at the same time. Ge et al. [16] proposed a vibration signal processing method combining WPT and EMD to extract time and frequencydomain features, applied them in the research of mechanical fault diagnosis, and achieved better results. After the original vibration signal is decomposed and demodulated by a certain vibration signal processing method, it becomes simple and intuitive. From its corresponding frequency spectrum, each frequency component can be clearly observed, so it is easier to extract useful state information from it. Some scholars combine the two modern vibration signal methods of EMD and wavelet packet decomposition. After denoising the original vibration signal through wavelet packet decomposition, the vibration signal is decomposed by EMD to extract highquality features [17-19]. Some scholars combined LMD and discrete wavelet transform (DWT) to modern vibration signal processing tools, proposed a new vibration signal decomposition method, and applied it to the fault diagnosis of rolling equipment; the vibration signal processing method not only inherits the adaptive characteristics of LMD but also uses 


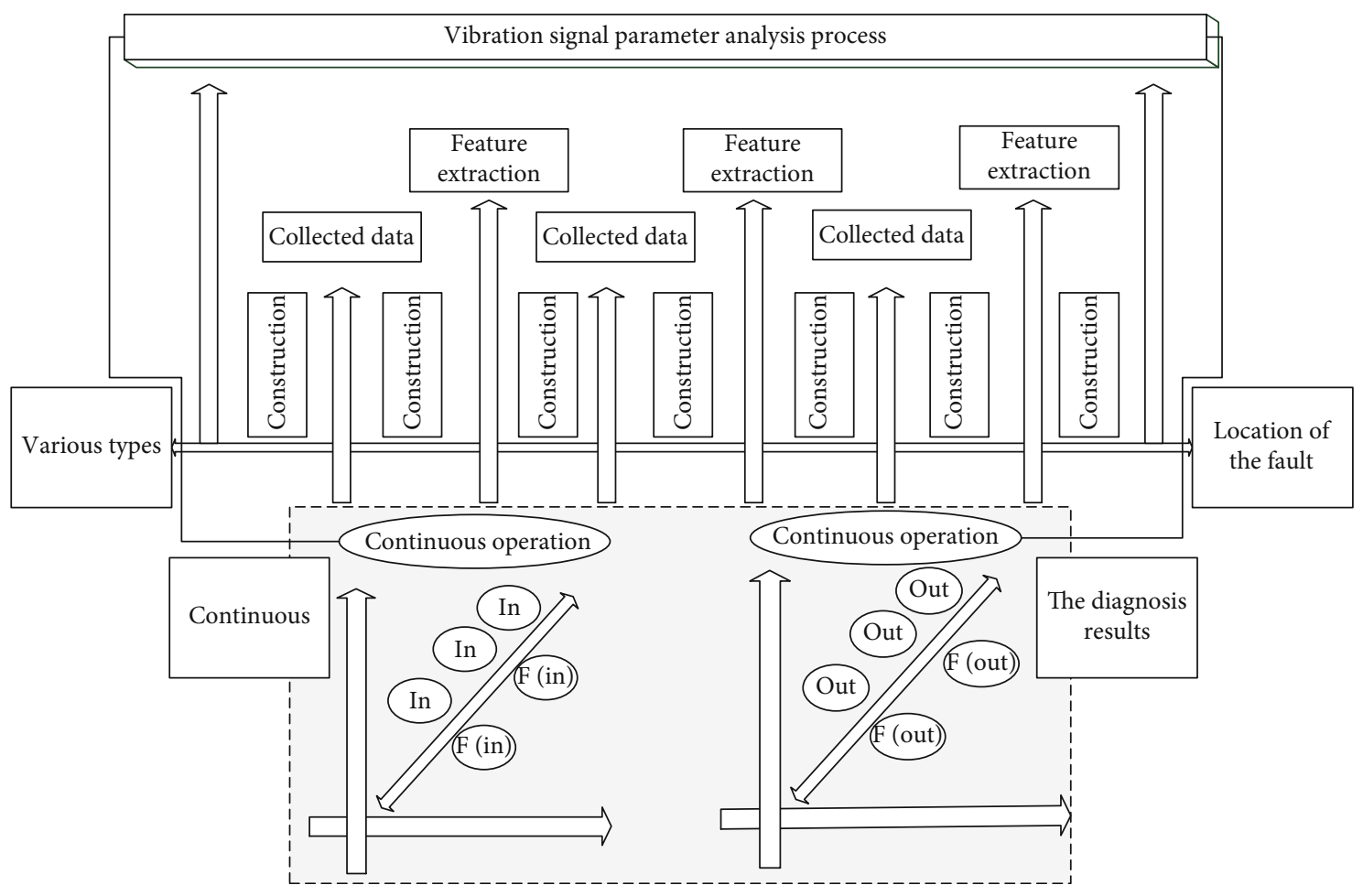

FIgURE 1: Vibration signal parameter analysis process.

discrete wavelet transform to minimize the modal aliasing problem of the LMD method [20,21].

\section{Construction of Life Prediction Model for Remanufactured Machinery and Equipment Based on Vibration Signal Feature Extraction}

3.1. Analysis of Vibration Signal Parameters. Vibration analysis method is currently one of the most effective methods used in equipment monitoring [22-25]. Sensors are installed at appropriate positions inside the equipment base or cabinet, and portable data collectors or boards are used to collect equipment vibration signals. The regularly collected data is transmitted to the computer for analysis and processing using relevant software to judge the bearing status and faults. Figure 1 shows the vibration signal parameter analysis process.

Because vibration monitoring has many advantages, such as being suitable for equipment of various types, various variable working conditions, and in continuous operation, it can realize online monitoring and offline measurement; it can diagnose early weak faults and the cause, type, and location of the fault.

$$
\begin{aligned}
& X(t)=\{t \subseteq R \mid x(1), x(2), \cdots, x(t)\}, \\
& y(n)=A \times X(n)-t \times x(n-1) .
\end{aligned}
$$

The characteristic parameters of the vibration signal have clear physical meanings and correspond to different types of faults. The vibration signal processing methods are diverse, and the diagnosis results are intuitive, effective, and reliable; therefore, the application is extremely wide.

$$
\begin{gathered}
\overline{x^{2}}(t, n)-|x(t, n)|^{2}-2|\overline{x(t, n)}| \times \cos \alpha=0, \\
\left\{\begin{array}{l}
\frac{\alpha(x)-\alpha(x-1)}{\sum a(t) \times w(x)}=1, \\
\frac{\beta(x)-\beta(x-1)}{\sum b(t) \times w(x)}=1 .
\end{array}\right.
\end{gathered}
$$

At present, equipment monitoring and diagnosis analysis instruments and systems are mostly made based on the principle of vibration, and more than $90 \%$ of the literature on equipment condition monitoring, fault diagnosis, reliability analysis, and remaining life analysis uses vibration signals. All digital signals obtained through the data acquisition system are a series of discrete values.

$$
\alpha=(i, j, k)=\{i(1), i(2) ; j(1), j(2) ; k(1), k(2)\}, i, j, k \subseteq Z,
$$

$$
\frac{\partial u}{\partial x}+\frac{\partial v}{\partial y}+\frac{\partial w}{\partial z}=0
$$

When the equipment fails, the statistical characteristic parameters of the amplitude of the vibration signal will also change with its location and the size of the fault. Therefore, the analysis of the amplitude-domain statistics can be used to diagnose the fault. Next, we will study several commonly 
used statistics describing the characteristics of vibration signals in the time domain.

$$
\begin{aligned}
& \left\{\begin{array}{l}
\phi(i, 1)=\frac{\phi^{+}(i)+\phi^{-}(i)}{2}, \\
\phi(i, 2)=\frac{\phi^{+}(i)-\phi^{-}(i)}{2},
\end{array}\right. \\
& \sum_{i}|\langle\alpha(1), u(i)\rangle|^{2}+\sum_{j}|<\alpha(2), u(j)>|^{2}=|\langle u(i, j)\rangle|^{2} .
\end{aligned}
$$

After the mechanical equipment is subjected to various external loads during the working process, the parts will be damaged, and the appearance of the damage will cause the change of the output parameters of the equipment.

$P(n, t)$ represents the value within the time interval $(n$, $n+1)$. Suppose that $z(f)$ represents the influence of external load, $u(f)$ represents the relationship of damage degree with time, and $x(t)$ represents the relationship of equipment output parameters with time. Damage is the characterization of the microscopic physical process in the equipment material, and the output parameter is the macroscopic reflection of the change process of the equipment health status. If the quantitative functional relationship between $x(f)$ and $U(f)$ can be determined, a model can be established based on the failure mechanism to perform quantitative research on the health of machinery and equipment.

$$
\begin{gathered}
\min T V(u)-\text { s.t. }\|\underset{u \in Z}{ } f-u(i, j)\|_{t}^{2}=\sigma^{2}, \\
\nabla \times p(x, t)+\nabla \times\left(\frac{\nabla u(x, t)^{k}}{\left|\nabla u(x, t)^{k}\right|}\right)=0 .
\end{gathered}
$$

The one-step prediction is based on $X$ as the input, that is, the prediction of the data at the next moment, using the actual observations of the previous $m$ consecutive moments. If in this process, the obtained predicted value is used instead of the true value, that is, no new information is added, the predicted value of the first data in the test set is obtained as the last component of the input vector for the next prediction to predict. At the next moment, this cycle will get a multistep forecast. In order to freely change the frequency and time-domain interval, the wavelet basis function needs to be scaled. The scale transformation includes the expansion coefficient and the translation coefficient.

$$
\begin{aligned}
\widehat{s} & =\frac{1}{(k(1) \times k(2))} \times \sum_{i=0}^{k 1} \sum_{j=0}^{k 2} s(i, j) \times p(x, t), \\
\operatorname{cov}(X) & =E\left[X X^{T}-\left(E(X) E(X)^{T}\right)\right] .
\end{aligned}
$$

When the basis function is narrowed, the high-frequency vibration signal can be extracted, and when the basis function is extended to a wider range, the low-frequency vibration signal can be extracted. Under certain scales, it has a coincidence relationship with the vibration signal and multiplies it to get a larger value. Through this result, you can get how much frequency components the original vibration signal contains. The frequency components contained in the original vibration signal and their corresponding time windows can be clearly identified. The time-frequency power diagram effectively contains the relevant characteristics of the original vibration signal.

3.2. Data Feature Extraction Algorithm. Traditional timedomain and frequency-domain analyses methods are both based on the stationary theory and cannot effectively analyze nonlinear nonstationary vibration signals. However, the dynamic response of mechanical equipment due to failures is a nonstationary process, and its vibration signals are also nonstationary. For vibration signals, time-frequency analysis came into being, and the two-dimensional joint analysis of time and frequency was used to describe the change of the statistical characteristics of nonstationary vibration signals over time.

$$
\begin{aligned}
& E(f(x))=\frac{\sum_{i=1}^{n} w(t) * f(x(t))}{\sum_{i=1}^{n} w(t)} \\
& \int f(x) p(t \mid x) p(x) d x=n \int f(x) p(x \mid t) d x \\
& P(x)=\int P(B \mid A(x)) P(A(x)) d A(x) .
\end{aligned}
$$

In the low-frequency part of the vibration signal, the vibration signal is relatively smooth and has obvious timedomain characteristics. There are many frequency components in this part. The frequency resolution can be improved by reducing the time resolution. In the high-frequency part of the vibration signal, it contains many characteristics of transient changes, and the relative frequency change has little effect on the vibration signal.

The wavelet transform decomposes the vibration signal into different scales (frequency domain) for description through operations such as expansion and translation and realizes the multiscale analysis of the vibration signal. Feature selection and feature transformation are a very important part of data-driven methods. After the feature extraction is completed, the performance of the degraded features is evaluated, and the best features are selected as the training input samples for the degradation state evaluation and remaining life prediction model. Through feature transformation, the core feature can be transformed to a few main variables to achieve the purpose of dimensionality reduction, thereby reducing the calculation amount of the model and improving the calculation efficiency of the model. Figure 2 shows the calculated efficiency histogram of the vibration signal model.

The various direct or indirect vibration signals monitored during the operation of mechanical equipment can be used to determine the working conditions of the mechanical equipment, and then, the future development trend of the mechanical equipment or its remaining life can be predicted. However, these vibration signals are the result of the nonlinear interaction of multiple factors, with nonlinear 


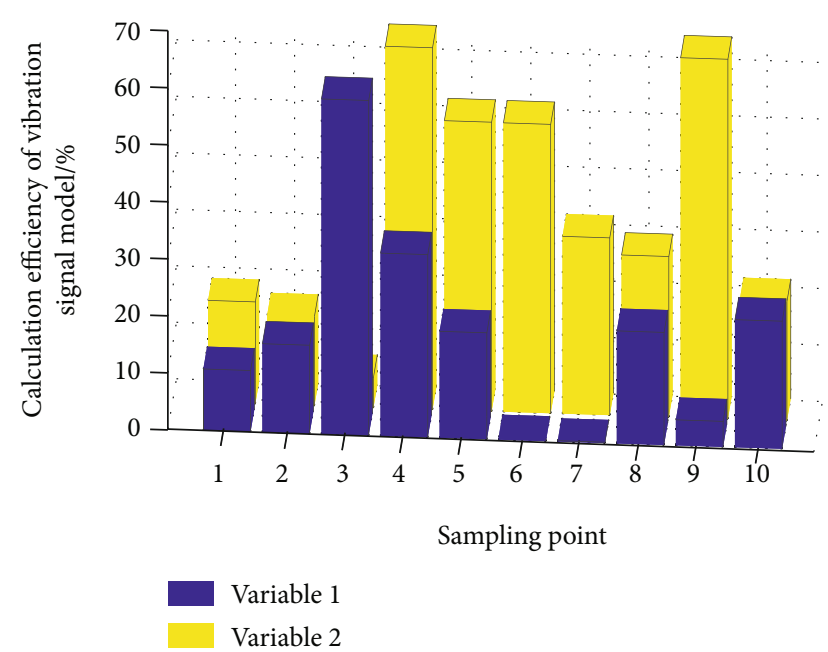

Figure 2: The calculation efficiency histogram of the vibration signal model.

characteristics such as chaos, classification, and irregularity, so the data has multiscale characteristics. The use of sensors can collect equipment status data in real time, which contains a variety of indications of the current status of the equipment. This information is contained in multiple feature spaces, while the vibration signal is only one-dimensional time-domain information, and only one-dimensional vibration is used.

First, after simple processing of the original data, continuous wavelet transformation is performed on the original vibration signals in the two directions, and the original time-domain information is extracted as time-frequencydomain two-dimensional image information. The image is fused and fused into six-channel feature data. ResNet is used to extract the degradation information contained in the image, and the time series features in the data are extracted through TCN, and the high-dimensional data is converted into a one-dimensional feature vector. Finally, the degradation process is linearly regressed. When a single fault occurs in the equipment, the time-domain characteristics can effectively describe its status information, but the evaluation effect for compound faults is not very good. Therefore, it is necessary to take the frequency-domain statistical characteristics into consideration. When the equipment fails, the energy of a specific frequency component on the spectrum will increase, and the entire spectrum distribution will be very different from before the failure. The frequencydomain characteristics can well show the information of the equipment's operating status.

3.3. Estimation of Mechanical Equipment Performance. The requirement of mechanical equipment failure prediction technology is to use all kinds of information generated during the working process of the equipment to comprehensively, accurately, and automatically determine whether the equipment is operating normally at any time, or the mechanical equipment has an abnormal sign, and accurately determine the cause and reason of the possible failure. When the equipment has a fault condition such as increased wear, lack of lubrication, burnout, or excessive load, it will cause the temperature at the equipment seat (or box) to change, and abnormalities can be found through temperature monitoring. However, when the equipment has relatively minor faults such as pitting, spalling, and slight wear, the temperature rise is not obvious, and only when the severity reaches a serious level, will there be a significant temperature rise.

In order to obtain the prior distribution of model parameters, it is necessary to solve the hyperparameters in the prior distribution, which can be obtained by distribution fitting. For the equipment to be tested, the measurement method can be selected according to the actual working environment. On the one hand, temperature sensors such as thermocouples and thermal resistances can be used for measurement. On the other hand, a noncontact infrared measuring instrument can be used for infrared imaging. Equipment needs to develop an independent monitoring system and alarm system. During analysis, the location of the hot spot can be found by comparing and analyzing with the normal temperature, so as to find the location with serious fault.

Figure 3 shows the design of the mechanical equipment performance monitoring system. Fault diagnosis refers to the process of analyzing and researching a situation, state, or problem of the equipment, detecting and identifying the failure mode in the system or subsystem. The fault prediction requires that the future state of the equipment can be carried out based on reasonable methods and relevant data obtained. Equipment wear is a gradual process, the peak value and RMS value of the vibration signal will gradually rise, and the waveform of the vibration signal is irregular, showing strong randomness.

It can be diagnosed whether there is a fault based on the change of the peak value and the RMS value, and the change of its value can also be predicted as a basis for life prediction. On the one hand, due to repeated impacts of rolling elements and defective working surfaces, it will cause periodic low-frequency vibrations. The frequency can be calculated according to the shaft speed and the geometric dimensions of the equipment, and it is the characteristic frequency of the fault to judge the type and extent of the fault by looking at the corresponding characteristic frequency and amplitude of the fault in the frequency spectrum of the vibration signal. On the other hand, the high-frequency natural vibration of the equipment system was caused by the impact, such as the radial bending natural vibration of the inner and outer rings of the equipment and the inherent vibration of the rolling elements.

3.4. Weight Analysis of Life Prediction. By setting the characteristic parameter monitoring threshold, it is assumed that this kind of early fault can be detected at a certain time after the "potential failure point of the component (fault detection point)." The purpose of fault prediction is to extract the basis of early fault detection and prediction feature information. On the above, according to the current component degradation state, we predict the time of failure, that is, the remaining life of the component. In a general sense, the period from "the current state point of the component" to the "function 


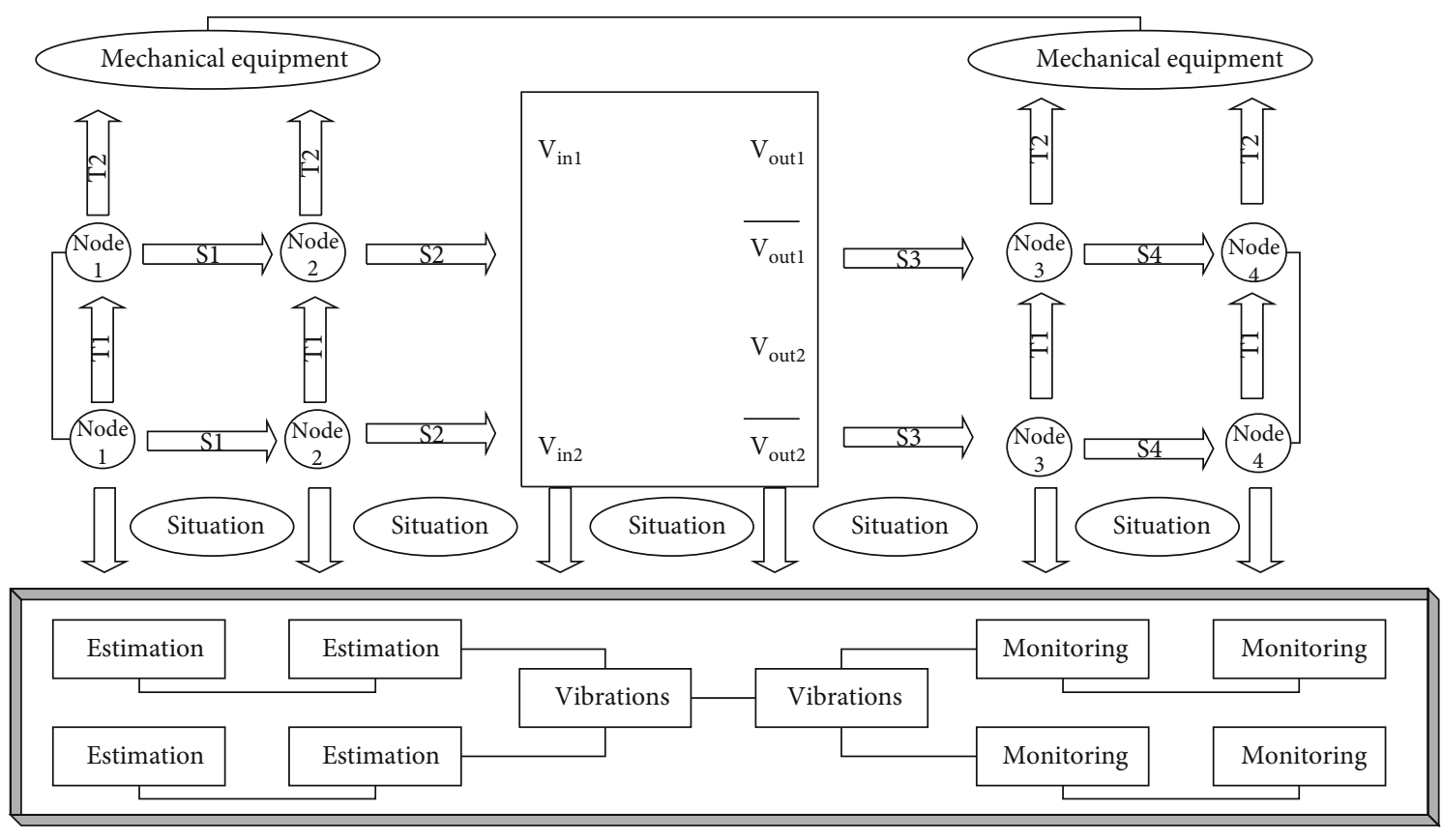

FIGURE 3: Mechanical equipment performance monitoring system design.

failure point of the component" is the remaining life of the component.

We test the probability that the normalized energy value exceeds the set threshold. Due to the influence of various factors, the remaining life of the mechanical equipment is not a fixed value, and we usually want to obtain the probability density function of the remaining life and then analyze the characteristics of equipment failure time. Time-domain analysis is to directly perform various calculations on vibration signals, and the calculation results still belong to the time-domain category. The time-domain statistical characteristic parameters are the low-order or high-order statistics of the time series, which are simple to calculate and have clear physical meanings. The statistical characteristic parameters of changes caused by vibration signals of different fault types and different fault levels are also different. For example, kurtosis and impulse indicators are more sensitive to shock faults, and the root mean square value is more effective for wear faults. Figure 4 shows the time-domain characteristic curve of the vibration signal.

Generally speaking, the time-domain statistical feature quantity provides a global feature of the equipment health status, but the root cause analysis of the failure is limited. Frequency-domain characteristic analysis firstly transforms the time-domain vibration signal into frequency-domain vibration signal through Fourier transform or Hilbert transform and then performs various calculation analysis methods on it, such as amplitude spectrum analysis, power spectrum analysis, and cross spectrum analysis and refined spectrum analysis, etc. By analyzing the frequency components in the frequency domain and corresponding to the characteristic fault frequencies of equipment and other parts during operation, the fundamental fault source can be found.

In order to effectively extract the feature quantity, it can usually be divided into two steps: first, we select the relatively sensitive and reliable feature quantity including the time domain and the frequency domain and start from the original data space; then, the space matrix containing all the features is reduced by linear or nonlinear methods. This paper connects to use the variance value, the simplest unsupervised feature selection algorithm to select the initial feature parameter vector, and preferentially select the feature with larger variance as the candidate feature to form the fault feature vector; then, perform PCA on the fault feature vector reduction processing. The PCA method can extract the main elements of the fault feature vector, reduce the dimensionality of the feature vector, and use a few irrelevant principal components to characterize the fault feature, which not only extracts effective information but also simplifies the data, making the equipment diagnosis work greatly simplify.

\section{Application and Analysis of Remanufacturing Machinery Equipment Life Prediction Model Based on Vibration Signal Feature Extraction}

4.1. Vibration Signal Feature Extraction. In the experiment, two high-frequency acceleration sensors (DYTRN 30358) were installed on the outer surface of the equipment, respectively, to measure the acceleration of the equipment in the horizontal and vertical directions. When the acceleration sensor is in use, its sampling frequency is set to $25.6 \mathrm{kHz}$, the sampling interval is $10 \mathrm{~s}$, and the duration of each sampling is $0.1 \mathrm{~s}$, that is, 2560 acceleration data points can be obtained for each sampling. The temperature sensor (PT100) is installed near the acceleration sensor and also close to the outer surface of the equipment. Its sampling frequency is fixed at $10 \mathrm{~Hz}$, and the sampling type is continuous sampling. The data acquisition card (NI DAQCard-9174) 


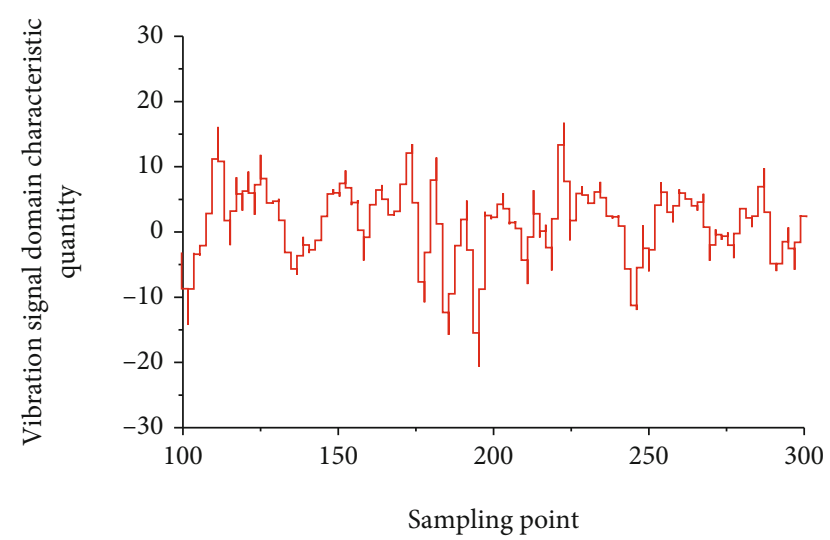

FIGURE 4: Time-domain characteristic quantity curve of vibration signal.

mainly includes three modules, which are used for two acceleration sensors, temperature probes, and torque sensors. When the acceleration amplitude exceeds $20 \mathrm{~g}$ continuously, the test is immediately terminated, and it is artificially concluded that the equipment has been significantly damaged at this time.

Figure 5 shows the interpolation line graph of vibration signal feature extraction. Starting from 1, every 15 samples belong to a state, and the sequence is as described above; the ordinate is the amplitude of the characteristic parameter. Since the physical meaning of each feature parameter is different, the scale of the parameter and the evaluation standard are also different. In order to evaluate each parameter and strengthen the classification performance of the classifier, each parameter is normalized. For different equipment failure states, the distinguishing ability of characteristic parameters is not the same and the rules are different. Some of the parameters are sensitive and reliable to the response law of the equipment state, such as the root mean square value; some parameters are not sensitive to the response of the equipment to different fault states, such as the mean value. It can be observed that after the Fast Fourier Transform (FFT) of the original vibration signal, there are many high-frequency interference components in the obtained spectrum. The original vibration signal undergoes wavelet threshold denoising, and most of the high frequency noise vibration signal in the frequency spectrum is successfully removed, leaving only a small amount of harmonic components.

Therefore, using the wavelet threshold denoising method to reduce the noise of the original vibration signal has achieved satisfactory results, which not only effectively removes the high-frequency interference information but also retains the useful information in the original vibration signal to a large extent. The vibration signal after noise reduction maintains a high signal-to-noise ratio, which is more conducive to further signal processing and analysis of the vibration signal.

4.2. Life Prediction Simulation of Mechanical Equipment. The rotating shaft is driven by the coupling of an AC motor and a friction belt, and a force of 32 is applied radially to the rotating shaft through a spring mechanism, and the equip- ment speed is constant at $2000 \mathrm{rpm}$. The equipment has 16 rolling elements in each row, the pitch circle diameter is 71.5 , the roller diameter is $8.4 \mathrm{~mm}$, and the contact angle is 15.17 .

The whole network contains 6 denoising automatic encoders, and the number of features extracted is 100 . In order to visualize the inherent change trend of the 100dimensional feature, considering the large difference in the range of each feature value, the principal component analysis of the 100-dimensional feature is carried out. From the process of the maximum principal component value changing with the sampling point, it can be seen that the noise is large. At the same time, the data acquisition software developed by PCB 353833 acceleration sensor and LabVIEW was used to collect the vibration signal at the equipment base. We calculate the root mean square (RMS) of the data every 10 minutes, and it can be seen that the equipment was in a normal state before the first 700 points. Therefore, the 780.930th point is used to train the support vector machine model, and the remaining 50 points are used to verify the model. Figure 6 shows the statistical distribution of the time sequence information of the vibration signal.

In the RUL prediction (remaining life prediction) stage, it is necessary to comprehensively consider the measurement data and historical data of the key equipment at the current moment and fully mine the time series information of the equipment operating data, so it is necessary to use a time series network to model the life prediction problem. Usually based on experience or using different wavelet bases to analyze the preprocessed data, we select the wave function that meets the requirements by comparing the analysis results. In digital vibration signal processing, Haar and Daubechies wavelet functions are usually selected. The compact support of the wavelet function means that the scale function and the wavelet function are only nonzero in a finite interval. The reliability distribution describes the probability that the RMS predicted value exceeds a set value, and $0.18 \mathrm{~A} /$ $\mathrm{g}$ is taken as the critical threshold of the RMS value.

In this paper, the bootstrap method is used to obtain the hyperparameters in the prior distribution of parameters. When the predicted value exceeds this threshold, we consider the equipment to fail. At the prediction point $N=40$, the RMS prediction value exceeds 0 . The probability of $18 \mathrm{~A} / \mathrm{g}$ is $2.41 \%$, but at the prediction point $N=45$, the probability of RMS exceeding the threshold is close to $100 \%$. According to calculations, at each prediction point $N=$ 40.45 , the probability that the RMS value exceeds the critical threshold is $2.41 \%, 8.62 \%, 29.31 \%, 66.55 \%, 94.13 \%$, and 99.5\%. According to the calculation results, it can be seen that as the number of predicted steps increases, the reliability gradually decreases. The first 150 data points are decomposed in three layers using $\mathrm{db} 3, \mathrm{db} 5$, and $\mathrm{db} 7$ wavelets and reconstructed by a single branch. It can be seen that as the length of the support increases, the reconstructed data curve of each layer is "cusp" reduce. The increase in the length of the wavelet function support makes the decomposed and reconstructed data curve become more "smooth," but this is also accompanied by an increase in the amount of calculation. 


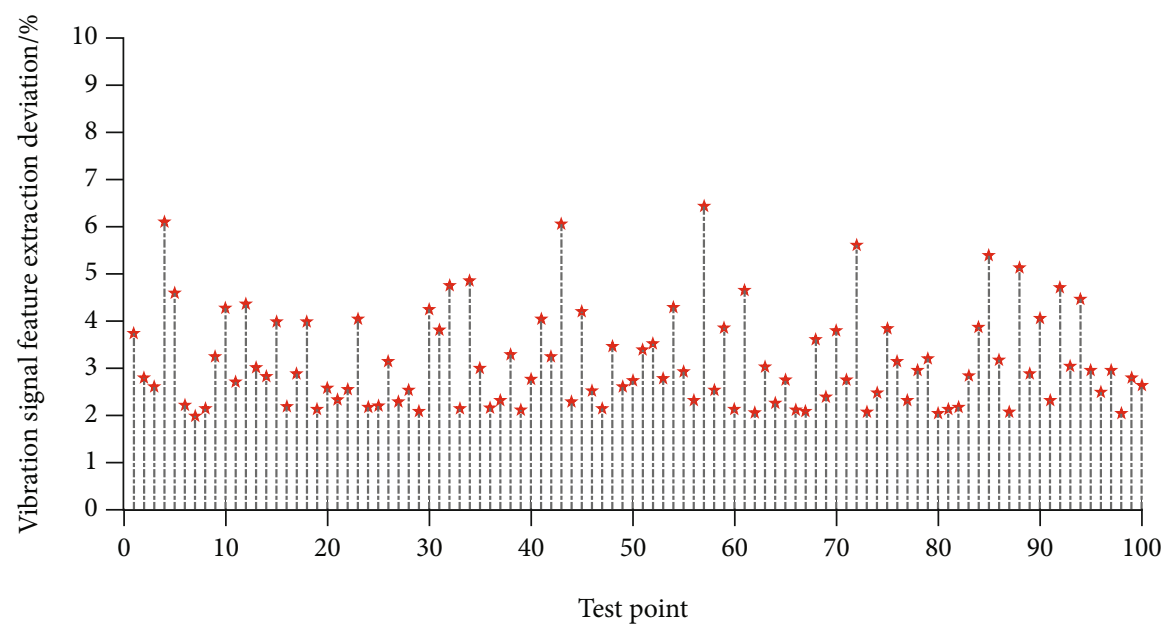

FIGURE 5: Interpolated line graph of vibration signal feature extraction.

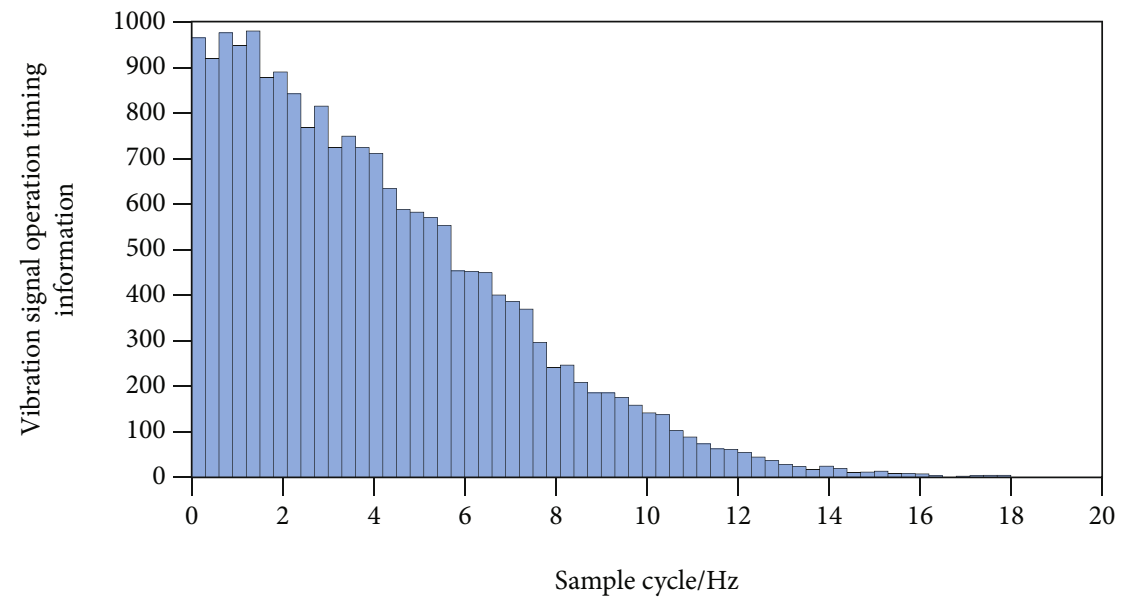

FIGURE 6: Statistical distribution of time sequence information of vibration signal operation.

4.3. Example Application and Analysis. In the modeling process of each decomposition sequence, the RBF kernel function is used, and the insensitive function is set to 0.01 . In order to estimate the model parameters, including the penalty factor $\mathrm{C}$ and the kernel function parameters, the grid method and the cross-validation method are combined. In the grid search, the search range is set to 1-100, and the search compensation is 1.0. The test set is divided into three parts for cross-validation, and the minimum mean square error value of each part is calculated separately, and then, the average value is calculated. Because there will be different parameters corresponding to the minimum mean square error, the minimum penalty factor $\mathrm{C}$ is regarded as the best combination of parameters. In the single support vector machine model, the penalty factor, the kernel function parameter, and the insensitive function value are $160,0.125$, and 0.01 . In the neural network model, the number of hidden layers is 10 , the number of output layers is 1, the input layer function takes the "tansig" function, and the "purelin" function is taken as the output layer function. The number of training times is 1000, and the training target error is 0.001 . Figure 7 shows the step diagram of the parameter accuracy deviation of the vibration signal model.

Under the same condition of model parameters, the predicted value of the first verification data obtained is used as the last component of the input vector of the next prediction to predict the next value. In this way, multistep prediction results can be obtained. Prediction error refers to the deviation between the predicted value of the model and the true value.

In order to analyze the prediction results of the above three models, this paper selects relative error (RE), mean absolute percentage error (MAE), and root mean square error (RMSE) as the judgment criteria. In the field of degradation state assessment and remaining life prediction, we are more concerned about the overall change trend of characteristics in the entire life cycle of the equipment. Since the input of the time series network training should be as consistent as possible with the test set, when the training data is specifically read, the data is not taken from the start running time of the training set equipment data, but 10,000 running moments are randomly selected from the training set. 100 continuous time-frequency power spectrograms before this 


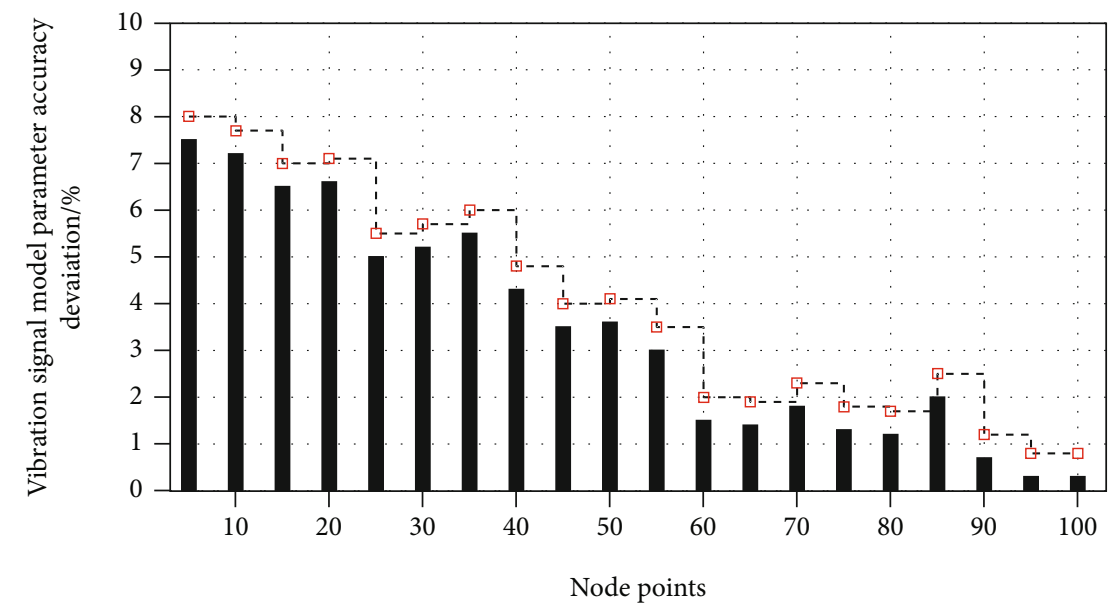

FIgURE 7: Ladder diagram of parameter accuracy deviation of vibration signal model.

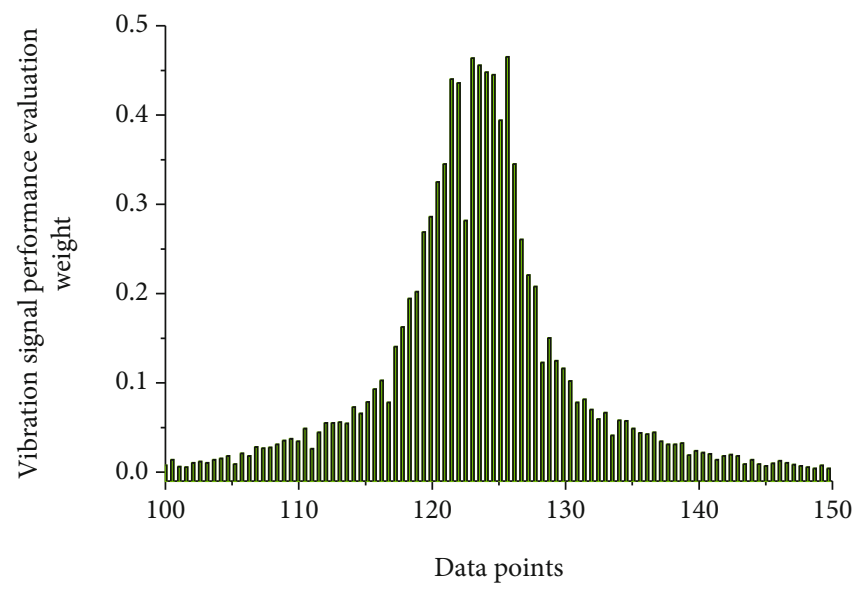

FIGURE 8: Histogram of vibration signal performance evaluation weights.

moment (including this moment) are sent to the pretrained ResNet network to generate training data. The last dimension is used as the output result, and the last real value of the health factor in each time window is selected as the label for training.

Figure 8 shows the histogram of the vibration signal performance evaluation weights. In this data set, each equipment has two sets of original vibration signals in the horizontal and vertical directions, the sampling frequency is $25.6 \mathrm{kHz}$, and 2560 sampling points (1/10 s) are recorded every $10 \mathrm{~s}$. The time-domain vibration data in both directions are shown in the text. Therefore, the monotonicity evaluation index of the degradation feature should get a relatively high importance weight. Here, we set the importance weights of the three evaluation indicators of relevance, monotonicity, and robustness to $0.2,0.55$, and 0.25 . Using the above feature selection method and analyzing the vibration data of the equipment, we can obtain the comprehensive index corresponding to the 96 features and the values of the three performance evaluation indexes. Their three characteristic evaluation indicators are all maintained at an upper-middle value, and then, the final comprehensive index $\mathrm{J}$ value is maintained at a relatively high position. Based on the construction of the degradation curve, the prediction model directly maps the equipment health value to the RUL value.

\section{Conclusion}

In the application example of the life prediction model, this paper measures the wear data during the equipment processing, uses the linear process with random drift effect to describe the change process of the equipment wear, and establishes the state space model. Combining the filtering algorithm, based on the vibration signal resampling, the unknown parameters of the model are obtained, and then, the degradation and remaining life of the equipment are predicted, and the optimal time is analyzed according to the corresponding decision-making model. Bootstrap method is an important estimation method in nonparametric statistics to estimate statistical variance and then carry out interval estimation. Aiming at predictable time-dependent failures of mechanical equipment, this paper studies a proportional failure model based on multiple decay characteristics, selects the characteristic quantities that reflect the equipment degradation trend as the input of the model, 
and finally determines the root mean square value and the wavelet packet sensitive energy value as the input covariate. The model is established based on the historical life and condition monitoring data of similar components, and then, the characteristic quantity predicted according to the own monitoring data is input into the model for real-time remaining life assessment, which realizes the real-time prediction of the remaining life of a single mechanical equipment, which is compared with the Logistic regression model. The research results show that, compared with the traditional mechanical equipment degradation state modeling method, the deep learning-based degradation state modeling method proposed in this paper can construct a smoother and less noisy performance degradation curve under both onedimensional and multidimensional monitoring data. And this method relies less on manual participation, the whole process is carried out in an unsupervised manner, and it has good versatility.

\section{Data Availability}

The data used to support the findings of this study are available from the corresponding author upon request.

\section{Conflicts of Interest}

The authors declare that they have no known competing financial interests or personal relationships that could have appeared to influence the work reported in this paper.

\section{Acknowledgments}

The study was supported by the 2021 Guangzhou science and Technology Project: Research on the key technology of reliability life prediction for remanufacturing equipment (No.: 202102080495) and 2021 Scientific Research Project of Education Department of Guangdong Province: Research on key technology of accurate positioning of underwater vehicle power system under uncertain factors (this project has no number).

\section{References}

[1] D. She and M. Jia, "A BiGRU method for remaining useful life prediction of machinery," Measurement, vol. 167, article 108277, 2021.

[2] X. Liu, Y. Jia, Z. He, and J. Zhou, "Application of EMD-WVD and particle filter for gearbox fault feature extraction and remaining useful life prediction," Journal of Vibroengineering, vol. 19, no. 3, pp. 1793-1808, 2017.

[3] B. Yang, R. Liu, and E. Zio, "Remaining useful life prediction based on a double-convolutional neural network architecture," IEEE Transactions on Industrial Electronics, vol. 66, no. 12, pp. 9521-9530, 2019.

[4] B. Zhang, L. Zhang, and J. Xu, "Degradation feature selection for remaining useful life prediction of rolling element bearings," Quality and Reliability Engineering International, vol. 32, no. 2, pp. 547-554, 2016.

[5] F. Chen, Y. Yang, B. Tang, B. Chen, W. Xiao, and X. Zhong, "Performance degradation prediction of mechanical equip- ment based on optimized multi-kernel relevant vector machine and fuzzy information granulation," Measurement, vol. 151, 2020.

[6] Y. Qin, S. Xiang, Y. Chai, and H. Chen, "Macroscopic-microscopic attention in LSTM networks based on fusion features for gear remaining life prediction," IEEE Transactions on Industrial Electronics, vol. 67, no. 12, pp. 10865-10875, 2020.

[7] H. Wang, G. Ni, J. Chen, and J. Qu, "Research on rolling bearing state health monitoring and life prediction based on PCA and Internet of Things with multi-sensor," Measurement, vol. 157, article 107657, 2020.

[8] D. Wang, K. L. Tsui, and Q. Miao, "Prognostics and health management: a review of vibration based bearing and gear health indicators," Ieee Access, vol. 6, pp. 665-676, 2018.

[9] X. Li, W. Zhang, and Q. Ding, "Deep learning-based remaining useful life estimation of bearings using multi-scale feature extraction," Reliability Engineering \& System Safety, vol. 182, pp. 208-218, 2019.

[10] M. Xia, T. Li, T. Shu, J. Wan, C. W. de Silva, and Z. Wang, "A two-stage approach for the remaining useful life prediction of bearings using deep neural networks," IEEE Transactions on Industrial Informatics, vol. 15, no. 6, pp. 3703-3711, 2019.

[11] F. Wang, X. Liu, G. Deng, X. Yu, H. Li, and Q. Han, "Remaining life prediction method for rolling bearing based on the long short-term memory network," Neural Processing Letters, vol. 50, no. 3, pp. 2437-2454, 2019.

[12] B. Wang, Y. Lei, N. Li, and T. Yan, "Deep separable convolutional network for remaining useful life prediction of machinery," Mechanical Systems and Signal Processing, vol. 134, article 106330, 2019.

[13] M. He, Y. Zhou, Y. Li, G. Wu, and G. Tang, "Long short-term memory network with multi-resolution singular value decomposition for prediction of bearing performance degradation," Measurement, vol. 156, article 107582, 2020.

[14] X. Li, W. Zhang, H. Ma, Z. Luo, and X. Li, "Data alignments in machinery remaining useful life prediction using deep adversarial neural networks," Knowledge-Based Systems, vol. 197, article 105843, 2020.

[15] B. Wang, Y. Lei, T. Yan, N. Li, and L. Guo, "Recurrent convolutional neural network: a new framework for remaining useful life prediction of machinery," Neurocomputing, vol. 379, pp. 117-129, 2020.

[16] Y. Ge, L. Guo, and Y. Dou, "Remaining useful life prediction of machinery based on KS distance and LSTM neural network," International Journal of Performability Engineering, vol. 15, no. 3, p. 895, 2019.

[17] Z. Meng, J. Li, N. Yin, and Z. Pan, "Remaining useful life prediction of rolling bearing using fractal theory," Measurement, vol. 156, article 107572, 2020.

[18] H. Yan, Y. Qin, S. Xiang, Y. Wang, and H. Chen, "Long-term gear life prediction based on ordered neurons LSTM neural networks," Measurement, vol. 165, article 108205, 2020.

[19] S. Xiang, Y. Qin, C. Zhu, Y. Wang, and H. Chen, "Long shortterm memory neural network with weight amplification and its application into gear remaining useful life prediction," Engineering Applications of Artificial Intelligence, vol. 91, article 103587, 2020.

[20] J. Deutsch and D. He, "Using deep learning-based approach to predict remaining useful life of rotating components," IEEE Transactions on Systems, Man, and Cybernetics: Systems, vol. 48 , no. 1, pp. 11-20, 2018. 
[21] T. Wang, Z. Liu, and N. Mrad, "Remaining useful life prediction for rolling bearings using EMD-RISI-LSTM," IEEE Transactions on Instrumentation and Measurement, vol. 70, pp. 112,2021

[22] W. Deng, J. Xu, H. Zhao, and Y. Song, "A novel gate resource allocation method using improved PSO-based QEA," IEEE Transactions on Intelligent Transportation Systems, pp. 1-9, 2020.

[23] X. Shang and X. C. Liu, "Analysis method of dynamics and structure optimization for sealing mechanism under reducing vibration condition," Proceedings of the institution of mechanical engineers, vol. 233, no. 2, pp. 348-362, 2019.

[24] W. Deng, S. Shang, X. Cai, Y. Zhou, H. Chen, and W. Deng, "Quantum differential evolution with cooperative coevolution framework and hybrid mutation strategy for large scale optimization," Knowledge-Based Systems, vol. 224, article 107080, 2021.

[25] X. Qin, Y. Shen, W. Chen, J. Yang, and L. X. Peng, "Bending and free vibration analyses of circular stiffened plates using the FSDT mesh-free method," International Journal of Mechanical Sciences, vol. 202-203, article 106498, 2021. 